\title{
CAPACIDAD DE GENERAR IMÁGENES MENTALES MOTORAS EN BAILARINES PROFESIONALES Y SUJETOS SEDENTARIOS
}

\author{
Diego Agudo-Carmona, PhD ${ }^{1,2}$; Alicia Daimiel-Yllera, PT ${ }^{1}$; Patricia Vaz-Torralba, $\mathrm{PT}^{1}$; Alba Paris-Alemany, MD 1,2,3,4; \\ Ferran Cuenca-Martínez, MSc ${ }^{1,2}$; Roy La Touche, $\mathrm{PhD}^{1,2,3,4}$ \\ 1. Departamento de Fisioterapia, Facultad de Ciencias de la Salud. Centro Superior de Estudios Universitarios La Salle, Universidad Autónoma de Madrid, \\ Madrid, España. \\ 2. Motion in Brains Research Group, Instituto de Neurociencias y Ciencias del Movimiento (INCIMOV), Centro Superior de Estudios Universitarios La Salle, \\ Universidad Autónoma de Madrid, Madrid, España. \\ 3. Instituto de Neurociencia y Dolor Craneofacial (INDCRAN), Madrid, España. \\ 4. Instituto de Investigación Sanitaria del Hospital Universitario La Paz (IDIPAZ), Madrid, España
}

\section{Correspondencia:}

Roy La Touche, PT, PhD Departamento de Fisioterapia. CSEU La Salle. Universidad Autónoma of Madrid. Calle La Salle, no 10, 28023 Madrid, España Teléfono: (+34) 917401980

Fax: (+34) 913571730 E-mail: Roylatouche@yahoo.es

\section{Conflicto de Intereses:}

Los autores declaran no tener ningún conflicto de intereses. Este proyecto no ha sido presentado en ningún evento científico

\section{Financiación:}

Los autores declaran no haber recibido financiación/compensación para el desarrollo de esta investigación.

Comité de Ética:

CSEULS-PI-130/2016.

DOI:

https://doi.org/10.37382/jomts.v2i1.18

Recepción del Manuscrito:

8-Abril-2019

Aceptación del Manuscrito:

2- Diciembre-2019

Licensed under: CC BY-NC-SA 4.0 (c)(1)(0) www.jomts.com

\section{RESUMEN}

Objetivo: El objetivo principal del presente estudio fue evaluar la capacidad de generar imágenes mentales motoras entre bailarines profesionales y sujetos sedentarios. El objetivo secundario fue evaluar la relación entre la capacidad de generar imágenes mentales motoras y la cronometría mental.

Diseño: Estudio observacional transversal.

Marco: Conservatorio Superior de Danza.

Participantes: 60 participantes fueron divididos en dos grupos: bailarines profesionales $(\mathrm{GB})(\mathrm{n}=30)$ y sujetos sedentarios $(\mathrm{GC})(\mathrm{n}=30)$.

Variables: La capacidad de generar imágenes mentales motoras se evaluó a través del cuestionario de imagen del movimiento (MIQ-R) así como de la cronometría mental. El nivel de actividad física se evaluó a través del cuestionario internacional de actividad física (IPAQ).

Resultados: En relación con la capacidad de generar imágenes mentales motoras, se encontraron diferencias significativas inter-grupo en todas las variables analizadas $(\mathrm{p}<.05)$. Adicionalmente, las correlaciones más fuertes se encontraron en el GB, donde se halló una asociación negativa moderada entre la capacidad de generar imágenes mentales motoras y la cronometría mental $(r=-.54)$.

Conclusión: En base a los resultados obtenidos, se sugiere que los bailarines profesionales, presentan una mayor capacidad de generar imágenes mentales motoras que los sujetos sedentarios, empleando, además, un menor tiempo en el desarrollo de la práctica mental.

Palabras Clave: Imaginería Motora, Cronometría Mental, Nivel de Actividad Física 


\section{INTRODUCCIÓN}

La Imaginería Motora (IM) se define como una habilidad cognitiva y dinámica que implica la representación de una acción, internamente, sin su ejecución real motora (Decety, 1996; Frenkel et al., 2014). El entrenamiento de IM provoca una activación de las áreas cerebrales relacionadas con la planificación y ejecución del movimiento voluntario de manera muy similar que cuando la acción se realiza de manera real (Bunno, Suzuki y Iwatsuki, 2015; Grèzes y Decety, 2001; Lotze et al., 1999; Luft, Skalej, Stefanou, Klose y Voigt, 1998; Stephan et al., 1995; Wilson y Knoblich, 2005). Lebon, Byblow, Collet, Guillot y Stinear (2012), argumentaron que la coincidencia en la activación cortical de dichas áreas entre la ejecución motora real y la práctica mental es un medio fiable para evaluar la calidad de la representación mental.

Los programas motores almacenados en los sistemas de memoria procedural permiten la generación de imágenes mentales motoras sin la necesidad de un estímulo externo aunque se ha demostrado que aportar una información visual previa a una tarea de imaginación facilita la misma y provoca una actividad neurofisiológica mayor que si se realiza de manera aislada (Sakamoto, Muraoka, Mizuguchi y Kanosue, 2009; Taube et al., 2015; Vogt, Rienzo, Collet, Collins y Guillot, 2013).

La activación del comando motor durante la práctica mental no da lugar a una ejecución real de un movimiento activo debido probablemente a un mecanismo inhibidor en la corteza motora primaria sobre las vías descendentes cortico-espinales (Guillot y Collet, 2005).

Por tanto, la IM permite la práctica de movimientos sin necesidad de realizarlos físicamente, y es por ello que se ha empleado ampliamente en el entrenamiento de habilidades técnicas tanto en atletas como en músicos, así como en la neurorehabilitación (Calmels, Holmes, Lopez y Naman, 2006).

En el campo de la práctica deportiva, numerosas investigaciones sugieren que la IM acelera y mejora el aprendizaje de habilidades motoras, así como el rendimiento deportivo. Además se ha demostrado también que la práctica mental mejora, a nivel psicosocial, la motivación y la confianza de los atletas, y donde a su vez, provoca una disminución de la ansiedad inherente a un evento competitivo (Guillot y Collet, 2008; Ridderinkhof y Brass, 2015).

Es por lo tanto, que la IM podría considerarse como un complemento al entrenamiento físico debido a que la combinación de ambos, ha demostrado ampliamente ser más efectivo respecto al rendimiento que el entrenamiento físico de manera aislado (Feltz y Landers, 1983).

La capacidad de generar imágenes mentales motoras, puede depender de varios factores como el tipo y complejidad de la tarea motora a imaginar, el nivel de actividad física, el tipo de imaginación, el esfuerzo mental dedicado a la práctica mental o el tiempo empleado (Decety, Jeannerod, Germain y Pastene, 1991; Di Corrado, Guarnera y Quartiroli, 2014; Peixoto Pinto, Mello Russo Ramos, Lemos, Domingues Vargas y Imbiriba, 2017).

Es por lo tanto que el objetivo principal del presente estudio fue evaluar la capacidad de generar imágenes mentales motoras entre bailarines profesionales y sujetos sedentarios. El objetivo secundario fue evaluar la relación entre la capacidad de generar imágenes mentales motoras y la cronometría mental.

\section{MÉTODOS}

\section{Diseño de estudio}

Un estudio observacional-transversal con muestreo no-probabilístico fue realizado para evaluar la capacidad de generar imágenes mentales motoras entre bailarines profesionales (GB) y sujetos sedentarios como grupo control (GC). El diseño siguió las recomendaciones internacionales para la publicación de los estudios observacionales (STROBE) (von Elm et al., 2008). Se obtuvo el consentimiento informado y por escrito, de todos los participantes antes de la inclusión. Todos los participantes recibieron una explicación sobre los procedimientos del estudio los cuales, fueron planificados bajo las normas éticas de la Declaración de Helsinki y fueron aprobados por el comité de ética del Centro Superior de Estudios Universitarios de La Salle. 


\section{Participantes}

Un muestreo no-probabilístico por conveniencia de 30 bailarines profesionales y 30 sujetos sedentarios, fueron reclutados entre enero y junio de 2017. Los participantes bailarines profesionales fueron reclutados del Conservatorio Superior Nacional de Danza de Madrid mientras que el GC, se obtuvo del Centro Superior de Estudios Universitarios La Salle y de la Comunidad de Madrid a través de medios y redes sociales, carteles, folletos y correos electrónicos.

Los criterios de inclusión para el GB fueron los siguientes: (a) bailarines profesionales, y (b) hombres y mujeres en edades comprendidas entre 18 y 30 años.

Los criterios de inclusión para el GC fueron los siguientes: (a) nivel de actividad física bajo o inactivo (menor a 600 METS), y (b) hombres y mujeres en edades comprendidas entre 18 y 30 años. El criterio de exclusión para el GB fue la presencia de una lesión musculoesquelética que requiriese el cese de la actividad física en el momento del estudio, mientras que para el GC fue presentar niveles de actividad física mayores a 600 METs.

Los siguientes criterios de exclusión fueron comunes en ambos grupos: (a) presencia de dolor en el momento del estudio, (b) haber realizado un entrenamiento previo de IM, (c) haber participado en un estudio similar anteriormente, y (d) falta de un completo entendimiento del idioma español.

\section{Procedimiento}

Tras haber consentido su participación, todos los participantes incluidos recibieron un cuestionario sociodemográfico para completar el día que se realizaron las mediciones, el cual contenía datos referentes a la edad, género y estado civil. Posteriormente, cada participante tuvo que completar una serie de medidas de autoinforme para evaluar el nivel de actividad física, la capacidad de generar imágenes mentales motoras y la cronometría mental. Primero, se entregó el cuestionario internacional de actividad física (IPAQ) para asegurar los criterios de inclusión del presente estudio y seguidamente, el cuestionario revisado de imagen del movimiento (MIQ-R), además evaluando el tiempo empleado en cada tarea mental del mismo.

\section{Variables principales}

\section{- Nivel de actividad fisica}

El nivel de actividad física fue objetivado mediante el cuestionario IPAQ el cual, para clasificar a los sujetos, traduce los datos obtenidos en relación con la actividad física realizada en la última semana, en METs. Basándose en esta estimación de los METs consumidos, el IPAQ divide a los sujetos en tres niveles de actividad física: baja, moderada y alta. Este cuestionario ha mostrado una validez aceptable para medir la actividad física total. Por lo tanto, las propiedades psicométricas del cuestionario se aceptaron para su uso en estudios que requerían la medición de la actividad física (Roman-Viñas et al., 2010).

\section{- Capacidad de generar imágenes mentales motoras}

Para evaluar la capacidad de generar imágenes mentales motoras se utilizó el cuestionario MIQ-R, el cual tiene cuatro movimientos repetidos en dos subescalas, una visual y una cinestésica. Tras realizar el movimiento de manera real se recoge el tiempo que se ha empleado para realizar la tarea mental, y la puntuación de cada ítem del MIQ-R se realiza mediante una escala de siete puntos, donde 1 indica "muy difícil de ver/sentir", y 7 indica "muy fácil de ver/sentir". Presenta un alfa de Cronbach aceptables, siendo 0.84 para el total de la escala, 0.80 para la subescala visual, y 0.84 para la subescala cinestésica (Campos y González, 2010).

\section{Variables secundarias}

\section{- Cronometría mental}

Utilizando un cronómetro, se registró el tiempo dedicado a realizar cada tarea del MIQ-R. El tiempo registrado corresponde al intervalo entre el comando para iniciar la tarea, dado por el evaluador, y la respuesta verbal de conclusión de la tarea, dada por el sujeto. La cronometría mental es una medida fiable y que ha sido utilizada ampliamente para recoger medidas objetivas de la capacidad de imaginar (Guillot y Collet, 2005; Malouin, Richards, Durand y Doyon, 2008; Williams, Guillot, Di Rienzo y Cumming, 2015). 


\section{Cálculo del tamaño muestral}

El tamaño de la muestra se calculó para detectar diferencias entre los grupos en relación con los resultados de la variable primaria (capacidad de generar imágenes motoras mentales). Para realizar el calculó previamente se realizó un estudio piloto con 10 participantes (cinco bailarines y cinco sujetos sedentarios), se realizó una comparación de la capacidad de generar imágenes motoras con el MIQ-R entre los dos grupos mediante una $t$ de Student, se encontraron diferencias estadísticamente significativas y un tamaño del efecto moderado de $d=0.77$. Utilizando una potencia estadística del $90 \%$ (1- $\beta$ probabilidad de error) y una probabilidad de nivel de error $\alpha$ de, 05 se generó un tamaño total de la muestra de 60 participantes (30 por grupo). El programa informático para el cálculo del tamaño de la muestra fue el $G^{*}$ Power 3.1.7 para Windows $\left(G^{*}\right.$ Power ${ }^{\circledR}$ por la Universidad de Dusseldorf, Alemania) (Faul et al., 2007).

\section{Análisis estadístico}

El paquete estadístico para ciencias sociales (SPSS 22, SPSS Inc., Chicago, IL, EE. UU.) fue el software que se utilizó para el análisis estadístico. El nivel de significación para todas las pruebas se estableció a $\mathrm{p}<$ .05 .

En el análisis de datos se ha utilizado estadística descriptiva para mostrar los datos de las variables continuas que se presentan como media \pm desviación típica (DT), intervalo de confianza (IC) del 95\% y frecuencia relativa (porcentaje). La prueba de $\chi$ cuadrado se utilizó para comparar las diferencias entre las variables categóricas (nominales). Debido a que cada grupo estaba formado por 30 participantes, se decidió no realizar las pruebas de normalidad y utilizar test paramétricos de acuerdo con el teorema central del límite. Se aplicó la $\mathrm{t}$ de Student para muestras independientes como prueba estadística para comparar las variables continuas entre ambos grupos. Se calculó el tamaño del efecto ( $d$ de Cohen) para las variables estudiadas. De acuerdo con el método de Cohen, el efecto fue considerado como pequeño (0.20 a 0.49$)$, medio (0.50 a 0.79$)$ y grande $(>0.8)$ (Cohen, 1988). La relación entre la capacidad de generar imágenes y tiempo de realizar esa tarea se analizó mediante el coeficiente de correlación de Pearson. Un coeficiente de correlación de Pearson superior a .60 indica una fuerte correlación, un coeficiente entre .30 y .60 indica una moderada correlación, y una coeficiente menor a .30 una baja correlación (Hinkle, Wiersma y Jurs, 1990).

\section{RESULTADOS}

La muestra total de estudio la conformaron un total de 60 participantes que fueron divididos de manera equilibrada en dos grupos ( 30 bailarines profesionales y 30 sujetos sedentarios) que cumplieron los criterios de inclusión. No se encontraron diferencias estadísticamente significativas en los datos sociodemográficos entre ambos grupos (Tabla 1).

\section{Capacidad de generar imágenes mentales motoras}

El estudio comparativo mostró diferencias significativas en todas las variables analizadas $(p<$ .05 ). Se obtuvieron diferencias entre ambos grupos en las puntuaciones de la subescala visual del cuestionario MIQ-R con un tamaño del efecto grande $(\mathrm{t}=-2.18 ; \mathrm{p}<$ $.05 ; d=-1.20)$ y de la subescala cinestésica con un tamaño del efecto moderado $(\mathrm{t}=-2.48 ; \mathrm{p}<.05 ; d=$ $0.63)$ así como en la puntuación total $(\mathrm{t}=-2.57 ; \mathrm{p}<$ $.05 ; d=-0.66)$. En relación con la cronometría mental, se obtuvieron diferencias significativas entre ambos grupos con un tamaño del efecto grande $(\mathrm{t}=3.61 ; \mathrm{p}<$ $.01 ; d=0.93)$ así como en la cronometría mental en la subescala visual $(\mathrm{t}=3.58 ; \mathrm{p}<.01 ; d=0.92)$ y cinestésica $(\mathrm{t}=2.96 ; \mathrm{p}<.01 ; d=0.76)$. También se hallaron diferencias entre los grupos con respecto al nivel de actividad física con un tamaño del efecto grande $(\mathrm{t}=-11.02 ; \mathrm{p}<.01 ; d=-2.84)$ (Tabla 2). En relación con el género, se encontraron diferencias en el grupo del GB, donde se halló que las mujeres tenían una mayor capacidad de generar imágenes mentales motoras que los hombres y en el GC se obtuvo que son los hombres los que tienen una mayor capacidad de imaginar que las mujeres, aunque dichas diferencias, no fueron estadísticamente significativas $(p>.05)$. 
Tabla 1. Datos Sociodemográficos.

\begin{tabular}{|c|c|c|c|}
\hline Variables & $\begin{array}{c}\text { GC } \\
(\mathrm{n}=30)\end{array}$ & $\begin{array}{c}\text { GB } \\
(n=30)\end{array}$ & p-valor \\
\hline Edad (años) & $23.57 \pm 2.52$ & $23.40 \pm 3.03$ & .818 \\
\hline Género (M/F) & $\begin{array}{c}15(50 \%) / \\
15(50 \%)\end{array}$ & $\begin{array}{c}15(50 \%) / 15 \\
(50 \%)\end{array}$ & 1.0 \\
\hline Estado Civil & & & .598 \\
\hline Soltero/a & $19(63.3)$ & $17(56.7)$ & \\
\hline Casado/a & $0(0)$ & $0(0)$ & \\
\hline $\begin{array}{l}\text { Relación } \\
\text { estable }\end{array}$ & $11(36.7)$ & $13(43.3)$ & \\
\hline Viudo/a & $0(0)$ & $0(0)$ & \\
\hline
\end{tabular}

\section{Análisis de correlaciones}

La Tabla 3 muestra el análisis de correlaciones de Pearson. Las correlaciones más fuertes fueron halladas en el GB, donde se encontró una asociación negativa moderada entre la capacidad de generar imágenes mentales motoras y el tiempo empleado en la práctica mental $(r=-.54 ; \mathrm{p}<.01)$. También se hallaron asociaciones negativas en el GC pero estas fueron débiles. Solamente en el GB se encontraron asociaciones negativas débiles-moderadas entre la capacidad de generar imágenes mentales motoras visuales y el tiempo empleado en la práctica mental ( $\mathrm{p}$ $<.05)$ y no, en el GC ( $>.05)$.

\section{DISCUSIÓN}

El objetivo principal del presente estudio fue evaluar la capacidad de generar imágenes mentales motoras entre bailarines profesionales y sujetos sedentarios. Los datos obtenidos sugieren que los bailarines profesionales, presentan una mayor capacidad de generar imágenes mentales motoras que los sujetos sedentarios.

Son numerosas las hipótesis que intentan dar respuesta a los hallazgos reportados en la literatura científica actual para poder explicar las diferencias obtenidas en la capacidad de generar imágenes mentales motoras entre atletas y no atletas. Una de ellas, sugerida por Goss, Hall, Buckolz y Fishburne (1986), es las diferencias que existen en el control motor y en la integración de la información sensoriomotora. En dicho estudio, evaluaron la capacidad de generar imágenes mentales motoras y obtuvieron que aquellos sujetos que presentaban mayor capacidad adquirían nuevos movimientos con menos intentos que los sujetos con menor capacidad de imaginar. En relación a esta hipótesis, Robin et al. (2007), obtuvieron que tenistas con mayor capacidad de imaginar tenían mayor precisión en la devolución del saque que los que tenían peor capacidad de imaginación mental motora.

En adición a esto, en una investigación llevada a cabo por Sacco et al. (2006), realizaron práctica física y mental en base a pasos básicos de tango. Su objetivo fue centrar la atención consciente y sensitiva en los movimientos del baile los cuales requieren de gestos motores específicos. Lo que encontraron fue que la práctica de una tarea motora específica, junto con un entrenamiento de la capacidad cinestésica en pasos básicos de tango, provocó una reorganización cortical en los esquemas motores, los cuáles son claves en el proceso de imaginación, modificando así, el procesamiento de la información sensoriomotora en el cerebro. Encontraron una actividad aumentada del giro precentral y del lóbulo parietal inferior las cuáles están ampliamente relacionados con la IM cinestésica.

Es por tanto que el entrenamiento físico provoca cambios neuroplásticos a nivel cortical mejorando tanto en el procesamiento de la información aferente sensoriomotora, como eferente, en relación con el control del movimiento de gestos motores aprendidos pudiendo ser claves para justificar las diferencias entre la capacidad de generar imágenes mentales motoras entre atletas y no atletas.

En el estudio de Di Corrado et al. (2014), compararon la capacidad de generar imágenes mentales entre atletas karatecas, bailarines y sujetos sedentarios. Los hallazgos que obtuvieron fueron que ambos grupos de atletas presentaron una mayor capacidad de generar imágenes mentales en comparación con el grupo de sujetos sedentarios.

Otra posible hipótesis, para explicar dichas diferencias, puede residir en tres características claves en la capacidad de generar imágenes motoras mentales: la vivacidad, la facilidad y la controlabilidad (Callow y Hardy, 2004). 
Tabla 2. Análisis comparativo entre los grupos.

\begin{tabular}{|c|c|c|c|c|}
\hline Variables & $\begin{array}{c}\text { GC } \\
(n=30)\end{array}$ & $\begin{array}{c}\text { GB } \\
(n=30)\end{array}$ & Diferencia de Medias (95\% IC) & $\begin{array}{c}\text { Tamaño del efecto } \\
\text { ( } d \text { de Cohen) }\end{array}$ \\
\hline MIQ-R & $41.20 \pm 7.28$ & $47.00 \pm 9.93$ & $-5.80(-1.29 \mathrm{a}-10.30)^{*}$ & -0.66 \\
\hline MIQR-C & $20.10 \pm 4.59$ & $23.33 \pm 5.51$ & $-3.23(-.61 \text { a }-5.85)^{*}$ & -0.63 \\
\hline MIQR-V & $21.20 \pm 4.03$ & $26.67 \pm 4.99$ & $-2.56(-.21 \mathrm{a}-4.91)^{*}$ & -1.20 \\
\hline $\mathbf{C M}$ & $34.42 \pm 13.35$ & $24.31 \pm 7.48$ & $10.10(15.70 \text { a } 4.51)^{* *}$ & 0.93 \\
\hline $\mathbf{C M}-\mathrm{V}$ & $17.89 \pm 7.63$ & $12.29 \pm 3.82$ & $5.59(2.47 \text { a } 8.71)^{* *}$ & 0.92 \\
\hline CM-C & $16.53 \pm 7.18$ & $12.02 \pm 4.25$ & $4.51(1.46 \text { a } 7.56)^{* *}$ & 0.76 \\
\hline IPAQ & $403.18 \pm 180.32$ & $12707.36 \pm 6108.33$ & $-12304.18(-10070.84 \text { a }-14537.52)^{* *}$ & -2.84 \\
\hline
\end{tabular}

${ }^{*} p<, 05 ;{ }^{* *} p<, 01$; IC: Intervalo de Confianza; GC: Grupo Control; GB: Grupo de Bailarines; MIQ-R: Cuestionario Revisado de Imagen del Movimiento; MIQR-C: Subescala Cinestésica; MIQR-V: Subescala Visual; CM: Cronometría Mental; CM-C: Cronometría Mental en la Subescala Cinestésica; CM-V: Cronometría Mental en la Subescala Visual; IPAQ: Cuestionario Internacional de Actividad Física.

Tabla 3. Análisis de correlaciones de Pearson

\begin{tabular}{|c|c|c|c|c|}
\hline Variable & Grupo & MIQ-R & MIQ-RV & MIQ-RC \\
\hline \multirow[t]{2}{*}{ CM } & GC & $-.48^{* *}$ & -.26 & $-.53^{* *}$ \\
\hline & GB & $-.54^{* *}$ & $-.49^{* *}$ & $-.53^{* *}$ \\
\hline \multirow[t]{2}{*}{ CM-C } & $\mathrm{GC}$ & $-.46^{*}$ & -.29 & $-.47^{* *}$ \\
\hline & GB & $-.55^{* *}$ & $-.51^{* *}$ & $-.53^{* *}$ \\
\hline \multirow[t]{2}{*}{ CM-V } & GC & $-.41^{*}$ & -.18 & $-.49^{* *}$ \\
\hline & GB & $-.45^{*}$ & $-.40^{*}$ & $-.44^{*}$ \\
\hline
\end{tabular}

${ }^{*} p<.05 ;{ }^{* *} p<.01$; GC: Grupo Control; GB: Grupo de Bailarines; MIQ-R: Cuestionario Revisado de Imagen del Movimiento; MIQR-C: Subescala Cinestésica; MIQR-V: Subescala Visual; CM: Cronometría Mental; CM-C: Cronometría Mental en la Subescala Cinestésica; CM-V: Cronometría Mental en la Subescala Visual. 
Las Se han hallado diferencias en la actividad cerebral, medidos en electroencefalografía, en comparación entre sujetos que imaginaban de manera más vivaz de aquellos que no hacían. Isaac y Marks (1994), demostraron que la vivacidad de la imaginación tenía implicación sobre el aprendizaje motor, mostrando cambios más significativos en aquellos sujetos presentaban una imaginación más vivaz. Roberts, Callow, Hardy, Markland y Bringer (2008) sugieren que los atletas de alto nivel presentan una capacidad mayor de realizar tareas de imaginación más vivaces. Se ha sugerido que la vivacidad podría reflejar los procesos de formación, mantenimiento y transformación de la imagen mental en los sistemas de memoria (Ranganath, 2006).

Con respecto a la cronometría mental, los bailarines profesionales emplearon un menor tiempo que los sujetos sedentarios en generar las imágenes mentales motoras.

En relación a los resultados obtenidos, Guillot y Collet (2005), sugirieron que las duraciones de los movimientos imaginados deberían ser similares a la duración de su ejecución real. Además, las influencias sobre la ejecución real también se aplican a la imaginación de gestos motores, donde se demostró que al colocar un peso en el brazo, este se mueve de forma más lenta y también se imagina con un tiempo mayor que sin el mismo (Papaxanthis, Schieppati, Gentili y Pozzo, 2002). Sin embargo, Parsons (1994) y Rieger (2012), demostraron que movimientos desconocidos, poco comunes o bien incómodos, pueden conducir a que existan diferencias entre el tiempo empleado entre la imaginación y la ejecución real de una acción. Estos hallazgos podrían correlacionarse con los datos obtenidos en el análisis de correlaciones del presente estudio donde la hipótesis, reside en que los movimientos incorporados en el cuestionario MIQ-R resultaran más sencillos, más conocidos y cómodos para los bailarines profesionales que para las personas asintomáticas sedentarias.

Este estudio presenta diversas limitaciones que deben tenerse en cuenta a la hora de sacar conclusiones con los resultados obtenidos. La primera limitación a considerarse es que desde el punto de vista temporal este es un estudio de diseño transversal con lo cual los resultados son interpretables para ese momento en concreto y con la condición inmediata de ese sujeto, consideramos que tiene mucho interés realizar estudios de tipo longitudinal para analizar los condicionantes en el tiempo que puedan modificar la capacidad de generar imágenes, por ejemplo, el aplicar un entrenamiento con imaginería motora a corto y medio plazo.

Esta investigación se ha realizado con bailarines de danza clásica y contemporánea, sin embargo, no se ha realizado más comparaciones para determinar si en una disciplina tienen mayor capacidad de generar imágenes motoras que otra. Por otra parte, sería también interesante evaluar en futuros estudios si sujetos que tienen mayor capacidad de generar imágenes motoras también tienen mejor control motor o mayor capacidad para realizar movimientos complejos.

Finalmente consideramos que tendría mucho interés realizar futuras investigaciones en donde se comparen la capacidad de generar imágenes entre bailarines profesionales y amateurs $\mathrm{o}$ entre bailarines profesionales y sujetos con nivel de actividad física alto.

\section{CONCLUSIÓN}

Es por lo tanto que, en base a los datos obtenidos, se sugiere que los bailarines profesionales, presentan una mayor capacidad de generar imágenes mentales motoras que los sujetos sedentarios.

Además, parece existir, en los bailarines profesionales, una asociación negativa entre la capacidad de generar imágenes mentales motoras y el tiempo empleado en el desarrollo de la práctica mental, siendo este menor cuanto mayor es la capacidad de imaginación.

Finalmente, los datos obtenidos también sugieren que el nivel de actividad física parece tener un impacto en la capacidad de generar imágenes mentales motoras.

Futuros estudios son necesarios para una mayor comprensión de los mecanismos neurocognitivos subyacentes a los hallazgos obtenidos en relación con la práctica mental. Los autores declaran no tener ningún conflicto de intereses. 


\section{FRASES DESTACADAS}

$>$ Los bailarines profesionales mostraron una mayor capacidad de generar imágenes mentales motoras que los sujetos sedentarios.

$>$ Los bailarines profesionales utilizaron un menor tiempo en generar las imágenes mentales.

\section{REFERENCIAS}

Bunno Y, Suzuki T, Iwatsuki H. Motor imagery muscle contraction strength influences spinal motor neuron excitability and cardiac sympathetic nerve activity. $J$ Phys Ther Sci. 2015;27(12):3793-8 DOI: http://dx.doi.org/10.1589/jpts.27.3793.

Callow N, Hardy L. The relationship between the use of kinaesthetic imagery and different visual imagery perspectives. $J$ Sports Sci. 2004;22(2):167-77 DOI: http://dx.doi.org/10.1080/026 40410310001641449.

Calmels C, Holmes P, Lopez E, Naman V. Chronometric Comparison of Actual and Imaged Complex Movement Patterns. $J$ Mot Behav. 2006;38(5):339-48 DOI: http://dx.doi.org/ 10.3200/JMBR.38.5.339-348.

Campos A, González MA. Spanish version of the revised movement image questionnaire (miq-r): psychometric properties and validation. Rev Psicol del Deport. 2010;19:263-73.

Cohen J. 1988. Statistical power analysis for the behavioral sciences, 2nd ed. Hillsdale, NJ: Lawrence Erlbaum Associates.

Di Corrado D, Guarnera M, Quartiroli A. Vividness and Transformation of Mental Images in Karate and Ballet. Percept Mot Skills. 2014;119(3):764-73 DOI: http://dx.doi.org/10.2466 /22.24.PMS.119c30z6.

Decety J. The neurophysiological basis of motor imagery. Behav Brain Res. 1996;77(1-2):45-52 DOI: http://dx.doi.org/ 10.1016/0166-4328(95)00225-1.

Decety J, Jeannerod M, Germain M, Pastene J. Vegetative response during imagined movement is proportional to mental effort. Behav Brain Res. 1991;42(1):1-5.

von Elm E, Altman DG, Egger M, Pocock SJ, Gøtzsche PC, Vandenbroucke JP, STROBE Initiative. The Strengthening the Reporting of Observational Studies in Epidemiology (STROBE) statement: guidelines for reporting observational studies. $J$ Clin Epidemiol. 2008;61(4):344-9 DOI: http://dx.doi.org/10.1016/j.j clinepi.2007.11.008.

Faul F, Erdfelder E, Lang A-G, Buchner A. G*Power: A flexible statistical power analysis program for the social, behavioral, and biomedical sciences. Behav Res Methods. 2007;39(2):175-91 DOI: http://dx.doi.org/10.3758/BF03193146.
Feltz DL, Landers DM. The effects of mental practice on motor skill learning and performance: A meta-analysis. J Sport Psychol. 1983;25-57.

Frenkel M, Herzig D, Gebhard F, Mayer J, Becker C, Einsiedel T. Mental practice maintains range of motion despite forearm immobilization: A pilot study in healthy persons. $J$ Rehabil Med. 2014;46(3):225-32 DOI: http://dx.doi.org/10.2340/16501977-1263.

Goss S, Hall C, Buckolz E, Fishburne G. Imagery ability and the acquisition and retention of movements. Mem Cognit. SpringerVerlag; 1986;14(6):469-77 DOI: http://dx.doi.org/10.3758/BF03 202518.

Grèzes J, Decety J. Functional anatomy of execution, mental simulation, observation, and verb generation of actions: a metaanalysis. Hum Brain Mapp. 2001;12(1):1-19.

Guillot A, Collet C. Contribution from neurophysiological and psychological methods to the study of motor imagery. Brain Res Rev. 2005a;50(2):387-97 DOI: http://dx.doi.org/10.1016/j.brainre srev.2005.09.004.

Guillot A, Collet C. Duration of Mentally Simulated Movement: A Review. J Mot Behav. 2005b;37(1):10-20 DOI: http://dx.doi.org/10.3200/JMBR.37.1.10-20.

Guillot A, Collet C. Construction of the Motor Imagery Integrative Model in Sport: a review and theoretical investigation of motor imagery use. Int Rev Sport Exerc Psychol. 2008;1(1):3144 DOI: http://dx.doi.org/10.1080/17509840701823139.

Hinkle DE, Wiersma W, Jurs SG. Applied Statistics for the Behavioral Sciences. Source J Educ Stat. 1990;15(1):84-7.

Isaac AR, Marks DF. Individual differences in mental imagery experience: developmental changes and specialization. $\mathrm{Br} \mathrm{J}$ Psychol. 1994;85:479-500.

Lebon F, Byblow WD, Collet C, Guillot A, Stinear CM. The modulation of motor cortex excitability during motor imagery depends on imagery quality. Eur J Neurosci. 2012;35(2):323-31 DOI: http://dx.doi.org/10.1111/j.1460-9568.2011.07938.x.

Lotze M, Montoya P, Erb M, Hülsmann E, Flor H, Klose U, Birbaumer N, Grodd W. Activation of Cortical and Cerebellar Motor Areas during Executed and Imagined Hand Movements: An fMRI Study. J Cogn Neurosci. 1999;11(5):491-501 DOI: http://dx.doi.org/10.1162/089892999563553.

Luft AR, Skalej M, Stefanou A, Klose U, Voigt K. Comparing motion- and imagery-related activation in the human cerebellum: a functional MRI study. Hum Brain Mapp. 1998;6(2):105-13.

Malouin F, Richards CL, Durand A, Doyon J. Reliability of Mental Chronometry for Assessing Motor Imagery Ability After Stroke. Arch Phys Med Rehabil. 2008;89(2):311-9 DOI: http://dx.doi.org/10.1016/j.apmr.2007.11.006.

Papaxanthis C, Schieppati M, Gentili R, Pozzo T. Imagined and actual arm movements have similar durations when performed under different conditions of direction and mass. Exp Brain Res. 2002;143(4):447-52 DOI: http://dx.doi.org/10.1007/s00221-0021012-1.

Parsons LM. Temporal and kinematic properties of motor behavior reflected in mentally simulated action. $J$ Exp Psychol Hum Percept Perform. 1994;20(4):709-30. 
Peixoto Pinto T, Mello Russo Ramos M, Lemos T, Domingues Vargas C, Imbiriba LA. Is heart rate variability affected by distinct motor imagery strategies? Physiol Behav. 2017;177:189-95 DOI: http://dx.doi.org/10.1016/j.physbeh.2017.05.004.

Ranganath C. Working memory for visual objects: Complementary roles of inferior temporal, medial temporal, and prefrontal cortex. Neuroscience. 2006;139(1):277-89 DOI: http://dx.doi.org/10.1016/j.neuroscience.2005.06.092.

Ridderinkhof KR, Brass M. How Kinesthetic Motor Imagery works: A predictive-processing theory of visualization in sports and motor expertise. $J$ Physiol. 2015;109(1-3):53-63 DOI: http://dx.doi.org/10.1016/j.jphysparis.2015.02.003.

Rieger M. Motor imagery in typing: effects of typing style and action familiarity. Psychon Bull Rev. 2012;19(1):101-7 DOI: http://dx.doi.org/10.3758/s13423-011-0178-6.

Roberts R, Callow N, Hardy L, Markland D, Bringer J. Movement Imagery Ability: Development and Assessment of a Revised Version of the Vividness of Movement Imagery Questionnaire. J Sport Exerc Psychol. 2008;30(2):200-21 DOI: http://dx.doi.org/10.1123/jsep.30.2.200.

Robin N, Dominique L, Toussaint L, Blandin Y, Guillot A, Her $\mathrm{M}$ Le. Effects of motor imagery training on service return accuracy in tennis: The role of imagery ability. Int J Sport Exerc Psychol. 2007;5(2):175-86 DOI: http://dx.doi.org/10.1080/1612197X.2007. 9671818.

Roman-Viñas B, Serra-Majem L, Hagströmer M, Ribas-Barba L, Sjöström M, Segura-Cardona R. International Physical Activity Questionnaire: Reliability and validity in a Spanish population. Eur J Sport Sci. 2010;10(5):297-304 DOI: http://dx.doi.org/10.1080/ 17461390903426667.

Sacco K, Cauda F, Cerliani L, Mate D, Duca S, Geminiani GC. Motor imagery of walking following training in locomotor attention. The effect of "the tango lesson." Neuroimage. 2006;32(3):1441-9 DOI: http://dx.doi.org/ 10.1016/j.neuroimage .2006 .05 .018
Sakamoto M, Muraoka T, Mizuguchi N, Kanosue K. Combining observation and imagery of an action enhances human corticospinal excitability. Neurosci Res. 2009;65(1):23-7 DOI: http://dx.doi.org/10.1016/j.neures.2009.05.003.

Stephan KM, Fink GR, Passingham RE, Silbersweig D, Ceballos-Baumann AO, Frith CD, Frackowiak RS. Functional anatomy of the mental representation of upper extremity movements in healthy subjects. J Neurophysiol. 1995;73(1):37386.

Taube W, Mouthon M, Leukel C, Hoogewoud H-M, Annoni JM, Keller M. Brain activity during observation and motor imagery of different balance tasks: An fMRI study. Cortex. 2015;64:102-14 DOI: http://dx.doi.org/10.1016/j.cortex.2014.09.022.

Vogt S, Rienzo F Di, Collet C, Collins A, Guillot A. Multiple roles of motor imagery during action observation. Front Hum Neurosci. Frontiers; 2013;7:807 DOI: http://dx.doi.org/10.3389 /fnhum.2013.00807.

Williams SE, Guillot A, Di Rienzo F, Cumming J. Comparing self-report and mental chronometry measures of motor imagery ability. Eur J Sport Sci. 2015;15(8):703-11 DOI: http://dx.doi.org/10.1080/17461391.2015.1051133.

Wilson M, Knoblich G. The Case for Motor Involvement in Perceiving Conspecifics. Psychol Bull. 2005;131(3):460-73 DOI: http://dx.doi.org/10.1037/0033-2909.131.3.460. 